\title{
Mapping Intellectual Structures and Dynamics of Detecting Research Fronts Domain
}

\author{
Jianhua Wang, Hong Li* \\ School of Economics and Management, University of Chinese Academy of Sciences, Beijing, China \\ Email address: \\ Wangjianhua16@mails.ucas.ac.cn (Jianhua Wang), lihong@ucas.ac.cn (Hong Li) \\ ${ }^{*}$ Corresponding author
}

\section{To cite this article:}

Jianhua Wang, Hong Li. Mapping Intellectual Structures and Dynamics of Detecting Research Fronts Domain. International Journal of Economics, Finance and Management Sciences. Vol. 5, No. 5, 2017, pp. 268-275. doi: 10.11648/j.ijefm.20170505.16

Received: August 17, 2017; Accepted: September 27, 2017; Published: October 27, 2017

\begin{abstract}
To date, very few research has been taken to quantitatively visualize the intellectual evolution of the field of detecting research fronts. Based on literature-searching analysis from the Thomson Reuters Web of Science as well as scientometrics mapping analysis, this important research topic is analyzed by techniques from informetric and bibliometric domains to detect its intellectual structures and dynamics. After data reduction and clean-up, 1841 articles published from 1991 to 2016 are downloaded, and two network analyses are conducted: a bibliometric approach (i.e. co-occurrence and co-citation network) and a complex network approach utilizing Chen's CiteSpace. Results are illustrated as the following: (1) growth of publications is divided into three stages: a stagnant phase (1991 - 2002), a takeoff phase (2003 - 2009) and a blooming phase (2010 - 2016); (2) most of the research institutions in this domain are concentrated in the United States, China, Japan and some developed countries in Europe. Chinese research in this area is mainly concentrated in Wuhan university, Chinese academy of science and Dalian university of technology; (3) publications of detecting research fronts mainly focus on the disciplines of Compute Science, Engineering and Information science library science; (4) we find three main academic communities in this domain, and they mainly focus on development and application of methods detecting research fronts; (5) methods detecting research fronts are classified into two categories: citations analysis and topic words analysis; (6) Scientific researchers began to frequently use the database for scientometrics research after 2012 and medical field began to use this approach to understand some of the frontier dynamics in the field in 2014.
\end{abstract}

Keywords: Research Fronts, CiteSpace, Bibliometric, Co-citation Network

\section{Introduction}

In recent decades, scientific activities are playing an increasingly important role not only in solving social problems but also as seeds of industrial innovations. With the increase of interdisciplinary research, the cooperation among disciplines is more and more common in scientific research activities, and emerging science topics are springing up. These emerging scientific themes are also called research fronts. Research fronts represent areas of cutting-edge study in specific fields. The concept of the research front was first introduced by Price [1] and has received wide attention in recent years. Different scholars have different definitions and interpretations to the concept of the research front. Garfield defined " co-citation literature and citations of their papers" as the frontier of the study; Small [2] and Griffith [3] believed that co-citation clustering characterizes the current active research field; Persson [4] argued that the difference between the research frontier and the knowledge base is: "From the perspective of bibliometrics, citations form the frontiers of researches, and the cited literatures constitute the knowledge bases"; And Morris [5] defined the frontier as a large number of articles that continue to be referenced by a fixed set of basic articles unrelated to time; Raam, moed and raan et al [6] defined a research front as "a series of related issues and concepts of concern to a group of scientific researchers"; Chen [7] defined the frontier as a set of concepts and their basic research problems.

At present, the definition of "research frontier" is roughly divided into three categories, corresponding to three different methods of literature measurement identification, including the co-cited analysis, bibliographic coupling analysis and 
co-word analysis. Understanding the latest developments of a field not only can help researchers to explore ideas, attain new research ideas and find solutions to the problems, but also is good for providing quantitative analysis basis and management decision support for the discipline development planning of national, regional and scientific institutions.

The detection of research fronts is a multidisciplinary field, which was paid attention until the 1990s. It is a branch of scientometrics whose history can be traced back to the 1960s, the American historian Price, published "science since Babylon", laid the foundation for this subject. The methods of detecting research fronts are also diverse. The scientific frontier detection can be achieved by two ways, one is the expert as the core, through meeting, communication and other methods, the different experts in the field, forming the final frontier vision and guiding scientific practice, this method has long time cycle, the use of human and material resources; The other is taking the literature as the core, using the scientific statistical method to explore the journal papers and works published by various scholars, explore the inherent laws and characteristics, and then reveal the internal structure of the subject and the direction of the next step, and finish the scientific frontier detection based on the literature.

This article is committed to understand the evolution of research front detection and discover academic communities and research fronts of it.

\section{Methods and Data}

Scientometrics mapping is a visual technique of informatics that quantitatively displays structural and dynamic aspects of scientific researches. In this research, two mapping approaches were applied: geospatial analysis and co-citation analysis. Co-citation analysis is based on the assumption that two references are often cited together and they are associated in some ways [8]. Geospatial analysis is usually visualized over a worldwide or thematic map based on related spatial attributes or geolocations [9]. CiteSpace is a main analytical tool in this article, which is a kind of information visualization software developed by professor Chen of Drake university in 2004. It is mainly based on the theory of co-citation analysis and pathfinder network scaling algorithm to calculate the collection of documents in a specific field, in order to explore the main path and knowledge inflection point of the domain or subject evolution, and then analyze the evolution mechanisms of the subjects and make a reasonable prediction on the frontiers of the subject development [10,11]. Liu and Gui [8] used this method to map intellectual structures and dynamics of transport geography research.

The Web of Science (WoS) core collection database was chosen as the source for literatures related to detecting frontier research. In this paper, the Web of Science Core Citation Database was extracted to retrieve data according to the strategy "Topics $=($ Detect* OR discover* OR identif* $)$ AND ("Research Front*" OR "emerging trend?" OR "research trend?" OR "emerging research")". After data reduction and clean-up, the finial sample contains 1841 papers, and the timeframe covers the years from 1991 to 2016.

\section{Spatiotemporal Distributions}

Since the 1990s, detecting research fronts has become a rapidly developing interdisciplinary field, and it has been paid attention by increasing academics. As shown in Figure 1, the number of its publications is increasing year by year, which is consistent with the significant exponential law of $\mathrm{y}=$ $2.7002 e^{0.1845 x}$ (model-adjusted coefficient $R^{2}=0.951$ ). In terms of temporal variations, the number of publications has climbed, multiplying by around 82 times and ranging from 3 in the initial period to 247 in the terminal period. Meanwhile, the number of citations has increased from 0 to 8,816 . Accordingly, yearly average citation also has increased rapidly from 0 to 35 . According to the chronological order, its growth of publications is wavy and can be divided into three stages: a stagnant phase (1991 - 2002) where the number of publications were unstable and few; a takeoff phase (2003 2009) where publications increased sharply; a blooming phase (2010 - 2016) that presented an exponential growth (Figure 1).

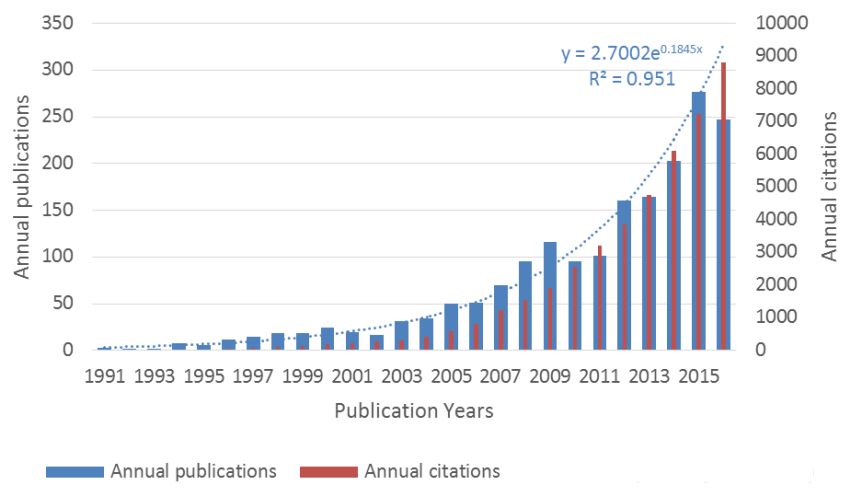

Figure 1. Historical Variations of Detecting Frontier Research Publications and Citations (1991 - 2016).

Using Google Earth of CiteSpace, global distribution maps of their institutes were generated (Figure 2). Results show that most of the research institutions are concentrated in the United States, China, Japan and some developed countries in Europe. The United States and some developed countries in Europe are established science and technology powers. They attach importance to the strategic layout of science and technology, and are more sensitive to the research frontiers of science. After world war II, Japan rose rapidly, so it also paid more and more attention to the strategic layout and frontier dynamics of science and technology. After the reform and opening up, the Chinese economy has developed rapidly. Chinese people were aware that science and technology are the first productivity. To develop science and technology, people must first understand the structures and frontier dynamics of science and technology, only in this way can researchers avoid detours. 


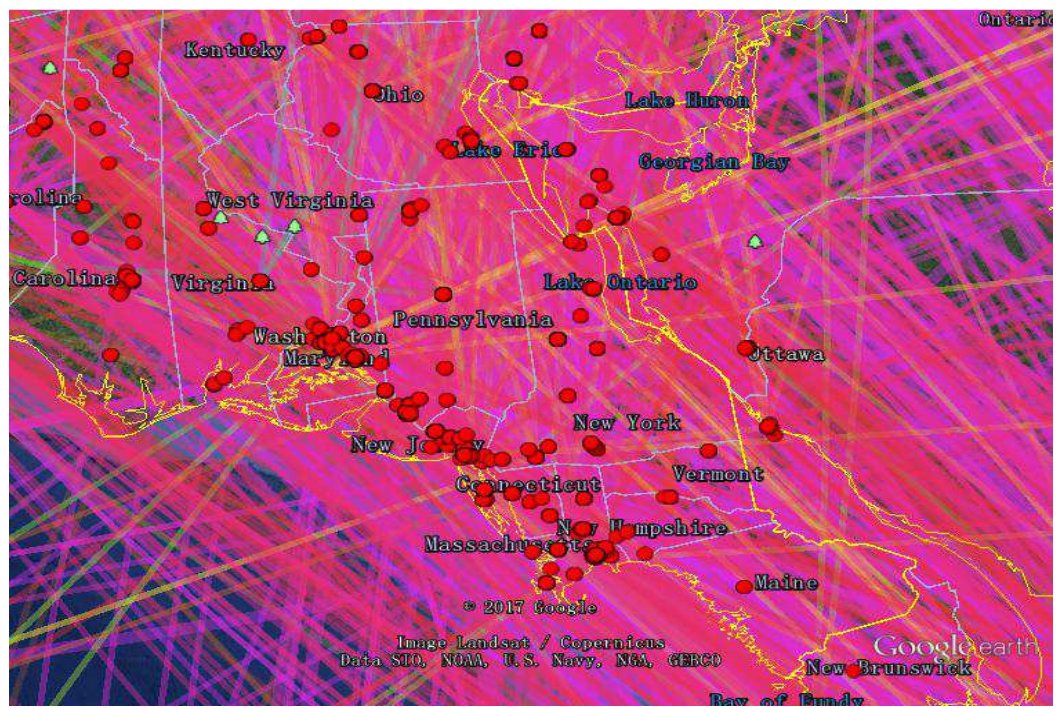

a. America

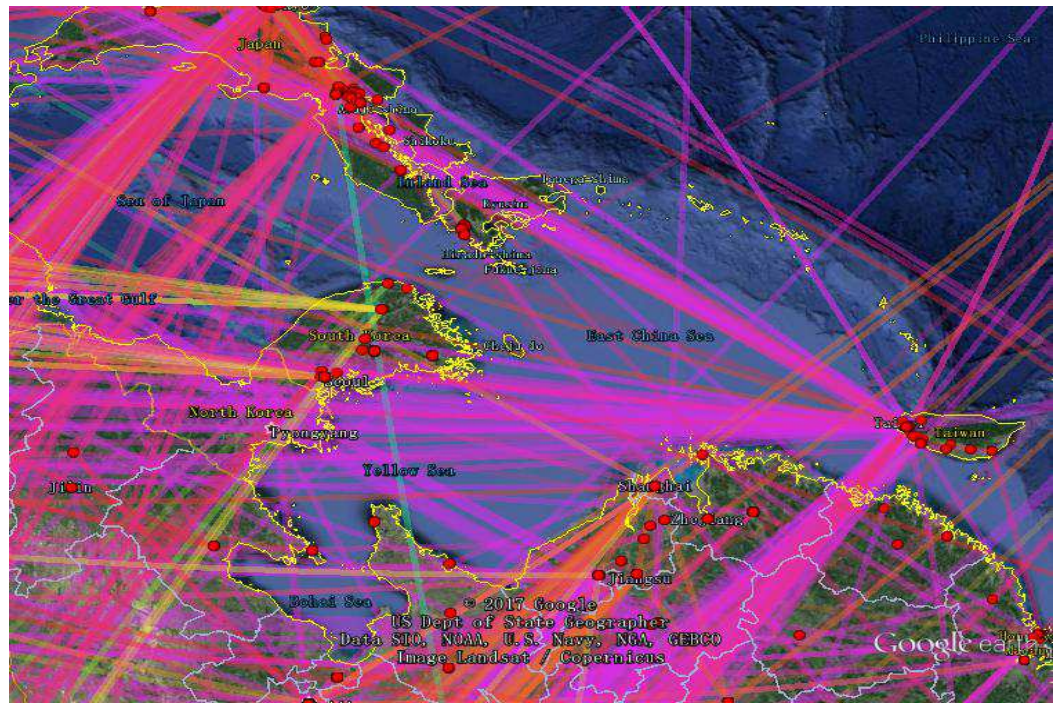

b. China and Japan

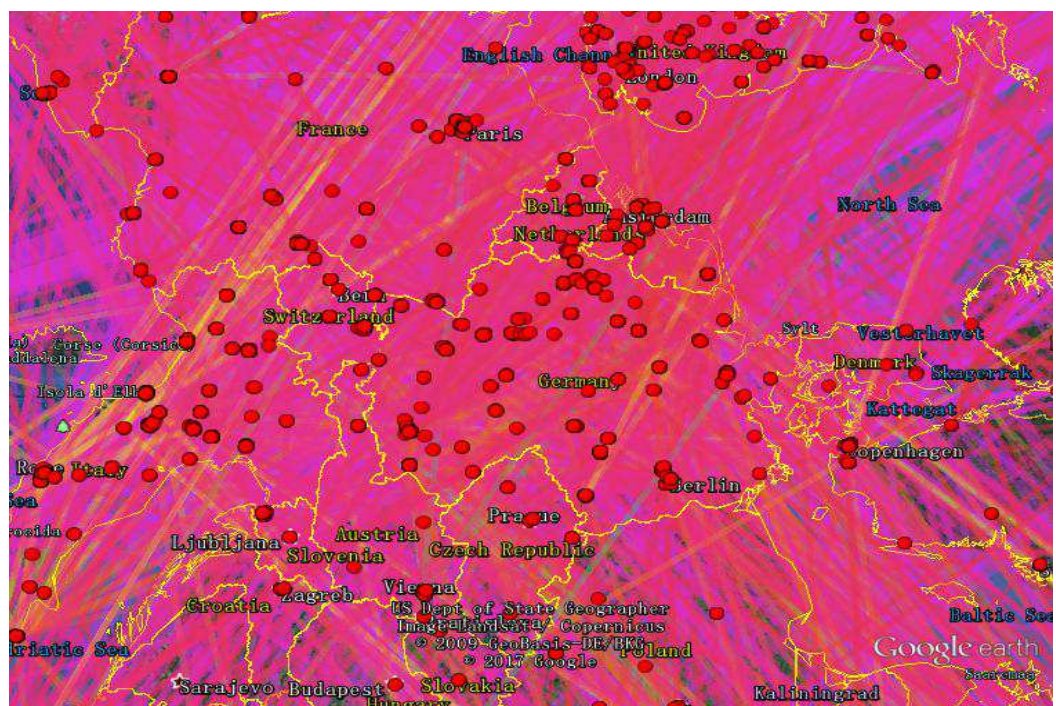

c. Europe

Figure 2. Distribution Map of Publications. 
By analyzing distribution of institutions, this research finds that 500 research institutions were involved in this field. As shown in Table 1, most of the research organizations are located in the United States, which shows that research strength of the United States in this field is very strong. It is worth mentioning that these are four Chinese research institutions in the top 40. They are WUHAN UNIVERSITY, CHINESE ACADEMY OF SCIENCES, DALIAN UNIVERSITY OF TECHNOLOGY and TSINGHUA UNIVERSITY respectively. Especially, WUHAN
UNIVERSITY ranks fourth. Professor Qiu Junping is mainly engaged in the teaching and research of library and information science, information management and evaluation in Wuhan university, and he has high attainments in bibliometrics and science. His work laid the status of Hanwu university in this field. In addition, Professor Liu Zeyuan of Dalian university of technology focuses on the teaching and research of science and technology management. The wise laboratory of Dalian university of technology has also made a great contribution to this field.

Table 1. List of Publication Organizations.

\begin{tabular}{|c|c|c|}
\hline NO & Organizations & Publications \\
\hline 1 & UNIVERSITY OF CALIFORNIA SYSTEM & 46 \\
\hline 2 & HARVARD UNIVERSITY & 36 \\
\hline 3 & UNIVERSITY OF NORTH CAROLINA & 28 \\
\hline 4 & WUHAN UNIVERSITY & 26 \\
\hline 5 & PENNSYLVANIA COMMONWEALTH SYSTEM OF HIGHER EDUCATION PCSHE & 25 \\
\hline 6 & NATIONAL INSTITUTES OF HEALTH NIH USA & 24 \\
\hline 7 & UNIVERSITY OF LONDON & 23 \\
\hline 8 & CENTRE NATIONAL DE LA RECHERCHE SCIENTIFIQUE CNRS & 22 \\
\hline 9 & UNIVERSITY OF PENNSYLVANIA & 20 \\
\hline 10 & UNIVERSITY OF MICHIGAN SYSTEM & 20 \\
\hline 11 & UNIVERSITY SYSTEM OF MARYLAND & 19 \\
\hline 12 & UNIVERSITY SYSTEM OF GEORGIA & 19 \\
\hline 13 & UNIVERSITY OF TOKYO & 19 \\
\hline 14 & UNIVERSITY OF MICHIGAN & 19 \\
\hline 15 & NATIONAL TAIWAN UNIVERSITY & 19 \\
\hline 16 & CHINESE ACADEMY OF SCIENCES & 19 \\
\hline 17 & UNIVERSITY OF PITTSBURGH & 18 \\
\hline 18 & STATE UNIVERSITY SYSTEM OF FLORIDA & 18 \\
\hline 19 & UNIVERSITY OF ILLINOIS SYSTEM & 17 \\
\hline 20 & UNIVERSITY OF BRITISH COLUMBIA & 17 \\
\hline 21 & INDIANA UNIVERSITY SYSTEM & 17 \\
\hline 22 & UNIVERSITY OF WASHINGTON SEATTLE & 15 \\
\hline 23 & UNIVERSITY OF WASHINGTON & 15 \\
\hline 24 & STATE UNIVERSITY OF NEW YORK SUNY SYSTEM & 15 \\
\hline 25 & DUKE UNIVERSITY & 15 \\
\hline 26 & UNIVERSITY OF WISCONSIN SYSTEM & 13 \\
\hline 27 & UNIVERSITY OF TORONTO & 13 \\
\hline 28 & UNITED STATES DEPARTMENT OF ENERGY DOE & 13 \\
\hline 29 & UNITED STATES DEPARTMENT OF DEFENSE & 13 \\
\hline 30 & DALIAN UNIVERSITY OF TECHNOLOGY & 13 \\
\hline 31 & UNIVERSITY OF SYDNEY & 12 \\
\hline 32 & UNIVERSITY OF ILLINOIS URBANA CHAMPAIGN & 12 \\
\hline 33 & TSINGHUA UNIVERSITY & 12 \\
\hline 34 & SEOUL NATIONAL UNIVERSITY & 12 \\
\hline 35 & OHIO STATE UNIVERSITY & 12 \\
\hline 36 & INDIANA UNIVERSITY BLOOMINGTON & 12 \\
\hline 37 & GEORGIA INSTITUTE OF TECHNOLOGY & 12 \\
\hline 38 & CONSEJO SUPERIOR DE INVESTIGACIONES CIENTIFICAS CSIC & 12 \\
\hline 39 & UNIVERSITY OF NORTH CAROLINA CHAPEL HILL & 11 \\
\hline 40 & DREXEL UNIVERSITY & 11 \\
\hline
\end{tabular}

\section{Layout of Research Areas}

Top 10 of the research areas are analyzed, which classified by the classification criteria of Web of Science (WOS). As shown in Figure 3, publications of detecting research fronts mainly source from Compute Science, Engineering and Information science library science. The detection of research fronts involves the development of many computing and visualization tools, and many research methods need to be 
realized by the algorithm. Therefore, the researches of this field cannot be carried on without the support of computer science. From the academic nature of the top 10 research areas, it can be known that the field is an interdisciplinary direction. From another angle, it can be also hold that detecting research fronts has been applied to a lot of different subjects. Research staffs understand the frontier dynamics of related disciplines, formulating the discipline development strategies and planning the discipline layout.

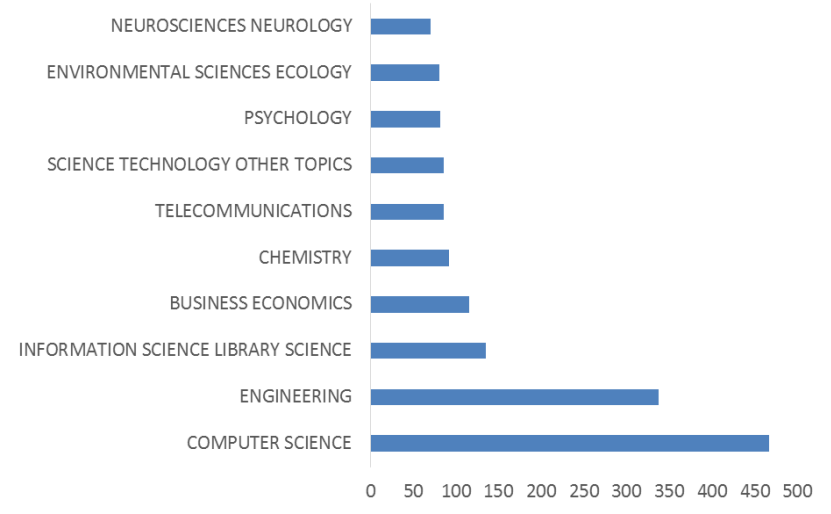

Figure 3. Distribution of Research Areas.

\section{Academic Communities}

Citation is the major indictor applied to measure paper's academic value. It is also the reflection of scientific achievements accepted by peers. Figure 4 is the network of author co-cited analysis based on CiteSpace, its nodes denote main authors and their achievements, and edges express their co-cited relationships. Size of vertexes represents co-cited degree of their works, and thickness of links represents co-cited frequents.

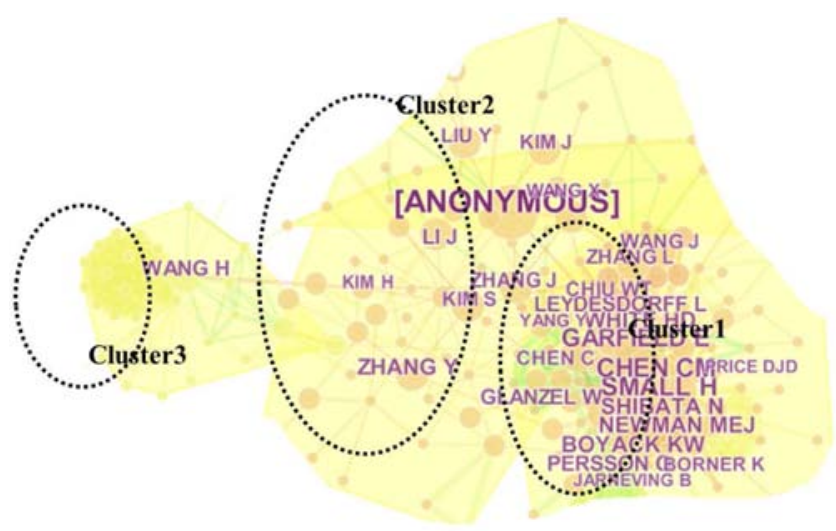

As shown in Figure 4, it can be found that three main clusters which represent different academic communities. Their research directions are various. There is not necessarily a fixed relationship between members of academic communities detected by us, but their researches are similar.

Label of cluster 1 is research. People in cluster 1 mainly focus on developing method detecting research fronts and mapping structure of subjects. For example, Garfield is the inventor of Science Citation Index (SCI), which provides the basis for the scientific researchers to study the structure, evolution and frontier of the discipline. Chen developed CiteSpace, which provides a convenient way for researchers to visual co-authorship networks, co-citation networks and the evolution of frontier trends. He used this tool to carry out many researches about the structure detection and evolution analysis of different subjects.

Label of cluster 2 is emerging trend detection, they principally concern on application of methods and theories. This cluster is mostly of Chinese people. It also shows that the Chinese people are in favor of application, and the researches on methods and theories are insufficient. Novelty of the research topics is critical for the competitiveness of research institutions and the development of science and technology. An, Lin, Yu and Zhang [12] proposed two new weighting methods to distinguish the emergence of research topics. Yang and Jung [13] focused on understanding scientific evolution by using keyword co-occurrence networks, using a change of power-law behavior to explore scientific evolution, such as emerging scientific paradigms and advancing normal science.

Label of cluster 3 is application, members of this cluster also pay attention to application. They analyzed the frontiers of researches, emerging hot spots and new trends in different fields. Abdallah, Maarof and Zainal [14] provided a systematic and comprehensive overview of these issues and challenges that obstruct the performance of Fraud detection system, and analyzed potential research trends of Fraud detection system in near future. As a new computing paradigm, cloud computing has received a lot of attention from enterprises and has being integrated or applied to enterprise architectures. Wang $\mathrm{H}, \mathrm{He} \mathrm{W}$ and Wang F-K [15] identify research trends and opportunities for researchers and practitioners in this field developing rapidly.

Figure 4. Network of Co-cited Authors.

Table 2. Academic Communities in Detecting Research Fronts.

\begin{tabular}{llll}
\hline Cluster ID & Size & Label & Main work \\
\hline 1 & 82 & research & Development of methods and theories \\
2 & 61 & emerging trend detection & Application and improvement of methods \\
3 & 29 & applications & Application of methods \\
\hline
\end{tabular}




\section{Classification of Methods Detecting Research Fronts}

The frontier detection methods are mainly divided into qualitative and quantitative analysis. Qualitative aspect, literatures review and Delphi method are more commonly used and more mature authority of the research frontier analysis methods. They are mainly inductive methods, collecting the first-hand data, starting from the personal background and knowledge accumulation, summing up, summarizing and refining different ideas, and finally forming a periodic review summary, current situation description or technology foresight, future prediction and so on, which can reflect the level of the subjects or the research levels and development trends. Quantitative detection and analysis have been the focus of researchers, not only because of the inherent defects of qualitative methods, but also because of quantitative methods in dealing with the analysis potential and common characteristics of large data.

Quantitative detection methods are divided into the following categories:

(1) Frontier detection methods based on citation network

Citation analysis is an important method and technology in the research of intelligence analysis, which has been widely used in the research of frontier detection. There are three types of citation relationships: direct citation, co-citation and bibliographic coupling. At present, the three citation relationships have been applied to the research fronts to some extent.

a. Detection method based on co-citation

Co-citation method, which refers to two or more papers cited in one or more subsequent papers at the same time, then the two quotations were regarded as co-citation literature.

Small was based on the thought of Price, expressing the frontier of scientific research as the same cluster of cited literatures. Small [16] proposed a co-citation clustering method to analyze the basic and early published core papers in a specific field. He believes that the co-citation clustering reflects features of the current active research areas.

The greatest challenge to the frontier identification of scientific research using co - citation analysis is delay. The reference information of the article needs a certain amount of time to accumulate.

b. Bibliographic coupling analysis method

The term "Bibliographic Coupling" was first proposed by M. M. Kessle, a professor at the Massachusetts institute of technology in the United States in 1963. When he carried out a citation analysis and research on the papers published in Physical Review, he discovered that the more similar the subject and the professional content, the more the number of the same documents in the reference literature.

Morris [5] added a timeline to the literature coupling clustering, and draws the research frontier time-line view. This view can show the emergence and disappearance of cutting-edge topics, potential emerging research frontier topics and their underlying documents.

Although the literature coupling analysis method overcomes the shortcomings of the delay of the common cited analysis method to some extent, the main problem encountered in the process when using the literature coupling to detect research fronts is the fixity of the data set.

c. Detection method based on network structure

In the study of complex networks, researchers found that many real networks have a common property, that is, community structure. In other words, the vertices in the network can be divided into groups, the connections among the vertices in the network are dense, and the connections between the vertices are sparse. In the scientific network, the community structure represents different research topics or research areas. Therefore, the community structure detection algorithm can be used to study the structure and evolution characteristics of science network. This is an emerging trend.

Trial optimization, spectral bisection, splitting, condensation and factional filtration are the five most representative research methods in the field of community detection in recent years, and the classical algorithms are following:

Kernighan [17] presented a heuristic method for partitioning arbitrary graphs which is both effective in finding optimal partitions, and fast enough to be practical in solving large problem, which is regarded as Kernighan-Lin algorithm; Pothen A [18] considered an algebraic approach for computing vertex separators, lower bounds on separator sizes can be obtained in terms of the eigenvalues of the Laplacian matrix associated with a graph by this. This method is recognized as spectral bisection method based on Laplace matrix; presented a method that allows for the discovery of communities within graphs of arbitrary size in times that scale linearly with their size. This method avoids edge cutting and is based on notions of voltage drops across networks that are both intuitive and easy to solve regardless of the complexity of the graph involved, which is regarded as Fast spectral segmentation method based on resistance network voltage spectrum; Girvan and Newman. [19] proposed a method for detecting such communities, built around the idea of using centrality indices to find community boundaries, and this method is regarded as GN algorithm; Existing algorithms detecting research fronts are computationally demanding, which limits their application to small networks. Newman [20] described an algorithm which gives excellent results when tested on both computer-generated and real-world networks and is much faster, typically thousands of times faster, than previous algorithms, which is regarded as Newman Fast algorithm.

(2) Frontier detection methods based on topic words

a. Burst Detection algorithm

Considering the emergence of a new scientific research frontier, it may be accompanied by the change of frequency density, Kleinberg [21] proposed a burst detection algorithm in 2002. The basic idea of the burst word detection algorithm is to observe the sudden change of words in a certain time and judge emergence of a new research topic according to the eruption of a large number of words. Professor Chen [7] used the Kleinberg burst word detection algorithm in the 
CiteSpace II software to obtain a cutting-edge topic description.

b. Detection method based on co-word

The research topic usually includes not only one or two subject headings, but a set of interrelated vocabulary. According to the relationship among words (i.e. the co-word relationship), this research clusters the words in the literature, and then a series of lexical clusters or semantic communities are generated, which also are called research topics. Thus, the existence of frontier areas can be revealed.

$\mathrm{Wu}$ and Zhang et al [22] integrated co-occurrence theory and clustering index to construct the topic segmentation model ATNLAD which is based on LDA probability topic model, digging into the literature theme and the relationship between them to explore the theme evolution law.

\section{Research Fronts}

Research fronts, originating from an emergent and transient grouping of concepts [1], mean the emerging thematic trends and surges of new topics, namely abrupt frequency increase of keywords from titles, abstracts, author keywords and keywords plus [7]. The sample data, after being imported into CiteSpace software and subjected to keyword co-occurrence analysis, generated 11 burst keywords. The co-occurrence network of keywords is shown as Figure 5 where the dark red circles represent the detected emergent keywords. Table 3 is the list of the top 11 Keywords with the Strongest Citation Bursts.

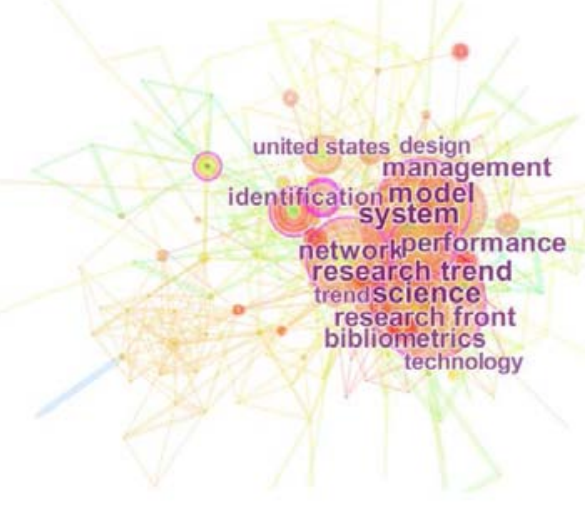

Figure 5. Co-occurrence Network of Keywords.

From Table 3, it can be found that the keyword gene expression started bursting in 2004, gene researches involve a variety of fields, which can help humans understand the nature of animals and plants and even human beings themselves. Understanding frontiers of this filed can be helpful for researchers to design and conduct researches. Furthermore, the keyword web of science burst out in 2012, which indicates that Scientific researchers began to frequently use the database for scientometrics researches after 2012. The web of science (WOS) database provides great convenience for researchers, and therefore promotes the rapid development in this field. With the development of database technologies, researchers said goodbye to the era of artificial computing, and began the computer era. This change allows researchers to analyze mass data and improve efficiency. Finally, the keyword randomized controlled trial is a method used in medicine, and it burst out in 2014. This shows that medical field began to use this approach to understand some of the frontier dynamics in the field, which helped medical researchers to better plan their own research programs and understand the novelty of their own researches.

Table 3. Top 11 Keywords with the Strongest Citation Bursts.

\begin{tabular}{llll}
\hline Keywords & Strength & Begain year & End year \\
\hline model & 4.6228 & 1995 & 2007 \\
gene expression & 4.0239 & 2004 & 2006 \\
web of science & 4.886 & 2012 & 2013 \\
bibliometric & 4.2356 & 2012 & 2014 \\
trend & 5.041 & 2013 & 2016 \\
perspective & 4.3541 & 2013 & 2016 \\
evolution & 4.2994 & 2013 & 2016 \\
research trend & 4.2565 & 2014 & 2016 \\
identification & 4.3162 & 2014 & 2016 \\
citation & 5.4462 & 2014 & 2016 \\
randomized controlled trial & 4.8592 & 2014 & 2016 \\
\hline
\end{tabular}

\section{Results and Discussion}

Despite being a subfield within scientometrics, a lack of the intellectual structures and evolution analyses of detecting scientific research fronts has been identified. To make up for this, big-data literature mining and scientometrics mapping have been used in this research to describe the spatial distribution of affiliations, layout of research areas, and academic community structures as well as to detect the emerging fronts.

From the point of time distribution, growth of publications in detecting emerging fronts domain is fluctuant and can be divided into three stages: a stagnant phase (1991 - 2002) where the number of publications were unstable and inadequate; a takeoff phase (2003 - 2009) where publications increased sharply; a blooming phase (2010 - 2016) that presented an exponential growth. The development of literature database technology has promoted the explosive growth of the third stage. From space distribution, the research institutions are mostly concentrated in the United States, China, Japan and some developed countries in Europe. The United States, Germany and France are the traditional technical powers, they attach importance to the strategic layout of science and technology, which requires understanding the structures and frontier dynamics of the disciplines to better conduct strategic planning and layout. China's researches on this area are mainly focused on Wuhan university, Chinese academy of science and Dalian university of technology. Since this field involves the knowledge of social networks, the construction and detection of the network requires the support of computer technology. Therefore, most articles in this field come from computer science.

By clustering communities, it can be found that there are three main academic communities in this domain, and they mainly focus on development and application of methods 
detecting research fronts. Through analyzing the content of literatures written by the members of academic communities, the methods detecting emerging research fronts are classified into two categories: citations analysis and topic words analysis. Citations analysis consists of co-citation analysis, Bibliographic coupling analysis and network analysis. Topic words analysis includes burst detection and co-word analysis. At present, there is no study to show which method is relatively effective. The researchers can select the appropriate method according to the needs of their own researches.

By analyzing the research fronts, it can be found that scientific researchers began to frequently use the database for scientometrics researches after 2012. Moreover, medical field began to use this approach to understand some of the frontier dynamics in the field in 2014.

This research still has some limitations, due to the inadequate quantity of publications in this field, the burst words detected are not too specific, leading to the failure to analyze deeply.

\section{References}

[1] D. D. Price, Networks of Scientific Papers, Science 149 (1965) $510-515$.

[2] Small, Co-citation in the Scientific Literature: A New Measure of the Relationship Between Two Documents, Journal of the American Society for Information Science 24(4) (1973) 265-269.

[3] Griffith, The Structure of Scientific Literatures II: Toward a Macro- and Microstructure for Science, Social Studies of Science 4(4) (1974) 339-365.

[4] Persson, The Intellectual Base and Research Fronts of JASIS 1986-1990, Journal of the American Society for Information Science 45(1) (1994) 31-38.

[5] Morris, Time Line Visualization of Research Fronts, Journal of the American Society for Information Science and Technology 54(5) (2003) 413-422.

[6] Braam, Mapping of Science by Combined Co-Citation and Word Analysis. I. Structural Aspects, Journal of the American Society for Information Science and Techology 42(4) (1991) 233-251.

[7] C. Chen, CiteSpace II: Detecting and visualizing emerging trends and transient patterns in scientific literature, Journal of the American Society for Information Science and Technology 57(3) (2006) 359-377.

[8] C. Liu, Q. Gui, Mapping intellectual structures and dynamics of transport geography research: a scientometric overview from 1982 to 2014, Scientometrics 109(1) (2016) 159-184.

[9] M. J. Cobo, A. G. López-Herrera, E. Herrera-Viedma, F. Herrera, Science mapping software tools: Review, analysis, and cooperative study among tools, Journal of the American Society for Information Science and Technology 62(7) (2011) 1382-1402.

[10] C. Chen, Searching for intellectual turning points: Progressive Know ledge Domain Visualization, Proceedings of the National Academy of Sciences of the United States of America 101 (2004) 5303-5310.

[11] C. Chen, F. Ibekwe-SanJuan, J. Hou, The Structure and Dynamics of Co-Citation Clusters: A Multiple-Perspective Co-Citation Analysis, Journal of the American Society for Information Science and Technology 61(7) (2010) 1386-1409.

[12] L. An, X. Lin, C. Yu, X. Zhang, Measuring and visualizing the contributions of Chinese and American LIS research institutions to emerging themes and salient themes, Scientometrics 105(3) (2015) 1605-1634.

[13] H. Yang, W.-S. Jung, Structural dynamics of keyword networks Liquid crystal display and plasma display panel cases, Journal of Engineering and Technology Management 40 (2016) 64-75.

[14] A. Abdallah, M. A. Maarof, A. Zainal, Fraud detection system: A survey, Journal of Network and Computer Applications 68 (2016) 90-113.

[15] H. Wang, W. He, F.-K. Wang, Enterprise cloud service architectures, Information Technology and Management 13(4) (2012) 445-454.

[16] H. Small, The Structure of Scientific Literatures I: Identifying and Graphing Specialties, Science Studies 4(1) (1974) 17-40.

[17] L. i. S. Kernighan An Efficient Heuristic Procedure for Partitioning Graphs Bell System Technical Journal 49(2) (1970) 291-307.

[18] S. H. Pothen A, PARTITIONING SPARSE MATRICES WITH EIGENVECTORS OF GRAPHS, SIAM J Matrix AnalAppl 11(3) (1990) 430-452.

[19] M. Girvan, Newman., Community structure in social and biological networks Proc. Natl. Acad. Sci 99 (2002) 7821-7826.

[20] Newman, Fast algorithm for detecting community structure in networks, Phys Rev E 69(6) (2004) 066133.

[21] K. J, Bursty and Hierarchical Structure in Streams, Data Mining and Knowledge Discovery 7(4) (2003) 373-397.

[22] Q. Wu, C. Zhang, X. An, Topic segmentation model based on ATNLDA and co-occurrence theory and its application in stem cell field, Journal of Information Science 39(3) (2012) 319-332. 